\title{
A PROSPECTIVE TRIAL COMPARING THE ACCURACY BETWEEN ULTRASOUND GUIDED FNAC OF AXILLARY LYMPH NODES AND SENTINEL LYMPH NODE BIOPSY USING METHYLENE BLUE FOLLOWED BY IMPRINT CYTOLOGY IN CLINICALLY NODE NEGATIVE EARLY BREAST CANCERS
}

\author{
M. Sivakami ${ }^{1}$
}

${ }^{1}$ Senior Consultant Pathologist, Department of Histopathology, Meenakshi Mission Hospital and Research Centre, Madurai, Tamilnadu, India.

\section{BACKGROUND}

Surgical treatment of breast cancer has evolved from Halsted's radical mastectomy to breast conserving surgery. Similarly, surgical treatment of regional lymph nodes has also become less extensive. Axillary lymph node dissection (ALND) was standard of care for a long time and considered necessary for loco-regional control as well as for staging purpose. While ALND came with substantial morbidity and the majority of the ALND patients were "node negative", various studies reported no effect of ALND on disease free and overall survival. In the mid-nineties the sentinel lymph node biopsy (SLNB) was introduced for staging in breast cancer patients. Conceptually, the search for sentinel lymph nodes (SLNs) in cancer patients was not new. In 1959 Gould presented his results of SLNB in patients treated for cancer of the parotid ${ }^{1}$ followed by a report describing the retrieval of SLNs in penile cancer. Two decades later Morton popularized the use of SLNB in melanoma patients. In 1994 it was Giuliano ${ }^{2}$, working in the same institute as Morton, who first described the use of the procedure in breast cancer patients. Nowadays this procedure is standard of care in clinically node negative breast cancer patients.

\section{MATERIALS AND METHODS}

FNA done after USG of breast and axilla followed by methylene blue injection. Blue nodes identified and harvested.

\section{RESULTS}

Ultrasound evaluation of node done, and suspected nodes documented. Methylene blue dye injected nodes were harvested. FNAC evaluation and imprint cytology evaluation of nodes done and compared with histopathology findings.

\section{CONCLUSION}

In our study, using methylene blue dye gave high accuracy in evaluation of positive lymph nodes. Imprint cytology is a useful adjuvant and is less expensive method to identify positive nodes than frozen section.

\section{KEY WORDS}

Breast Cancer, Sentinel Node Biopsy, Methylene Blue, FNAC/ Imprint Cytology

HOW TO CITE THIS ARTICLE: Sivakami M. A prospective trial comparing the accuracy between ultrasound guided FNAC of axillary lymph nodes and sentinel lymph node biopsy using methylene blue followed by imprint cytology in clinically node negative early breast cancers. J. Evolution Med. Dent. Sci. 2019;8(10):662-666, DOI: 10.14260/jemds/2019/147

\section{BACKGROUND}

The incidence of breast cancer with the Urban Indian population is $34 \%$ of all cancers and has become the commonest cancer is women. W. Halstead first described radical Mastectomy for operable breast cancers. Later Patchy, Madden, and Auchinelaus used various modifications on the Radical mastectomy procedure to reduce the cosmetic and functional morbidity. Later on, Keynes, Akkins et al, Mustakallio, Hayward and other introduced various breast conservation surgical techniques. (QUART) Quadrantectomy with axillary dissection and Radiotherapy was described with results by Veronosi et al.

'Financial or Other Competing Interest': None.

Submission 02-04-2018, Peer Review 17-02-2019,

Acceptance 25-02-2019, Published 11-03-2019.

Corresponding Author:

M. Sivakami,

Meenakshi Mission Hospital and Research Centre,

Lake Area, Melur Road,

Madurai-625107,

Tamilnadu, India.

E-mail: sivakami.saru@gmail.com

DOI: $10.14260 /$ jemds $/ 2019 / 147$

\section{(c) $(1)(\$$}

Currently the most followed surgical techniques for breast cancers are modified Radical mastectomy with or without Breast conservation surgery (BCS). Various factors like patient's desire, size of tumour to breast ratio, availability of Radiotherapy techniques, Contraindicative factors for conservative surgery and surgeon's technique plays a role in the choice of surgical technique.

The axillary lymph nodes are divided into three different levels as level I level II and level III pectorals minor muscle in used as a Land mark for this division.

Similarly, surgical treatment of regional lymph nodes has also become less extensive. Axillary lymph node dissection (ALND) was standard of care for a long time and considered necessary for loco regional control as well as for staging purposes. While ALND came with substantial morbidity and the majority of the ALND patients were "node negative", various studies reported no effect of ALND on disease free and overall survival.

Modern screening methods and increased self-awareness about breast cancer has made it possible to diagnose breast cancer at an early stage. Axillary lymph node status is one of the most important prognostic indicators in breast cancer. 
Routine axillary dissection does not benefit $70-80 \%$ of early breast cancer patients who are clinically node negative. Also, axillary lymphadenectomy results in significant morbidity like chronic lymph oedema of ipsilateral extremity (3-12\%), frozen shoulder syndrome and long-term sensory abnormalities.

A study by Kelly M. McMasters revealed that preoperative lymphoscintigraphy for breast cancer does not improve ability to identify axillary sentinel nodes. ${ }^{3}$ Various other studies done using feline model to asses lymphatic drainage by Jan $\mathrm{H}$ Wong et al also revealed the same. ${ }^{4}$

A promising alternative to assess axillary lymph node status in early breast cancer patients is Sentinel Lymph Node Biopsy (SLNB) and USG guided FNAC Both USG 5 guided FNAC and sentinel lymph node biopsy have been useful to avoid a morbid axillary dissection.

Careful evaluation of level I, II, III axillary lymph nodes including the axillary tail is very important while doing staging ultrasonography. For this the patient is placed in a supine position. The proximal arm is positioned upwards adjacent to the breast. The distal arm can be kept on the forehead or above the head. High frequency linear transducer in the range of $12 \mathrm{MHz}$ to $17 \mathrm{MHz}$ probe is used for evaluation. Sometime $9 \mathrm{MHz}$ probe is used for larger bulky patients for deeper penetration. The pectoralis major and minor is first visualized, the medial aspect of axilla, upper outer quadrant of breast, axillary tail should be carefully examined. Level I nodes are usually easily visualised. The level II nodes are usually beneath the pectoralis minor, and are not usually seen but when enlarged can be easily visualised.

Various morphological charecteristics ${ }^{6}$ are available to identify node involvement apart from the size and enlargement alone. There are narrowing of node hilum with cortical thickening. Bulge in cortical contour which is seen as focal eccentric cortical thickening. Loss of fat in the hilum. Contour of the node is also an important feature while differentiating malignant from benign nodes. Long to short axis ratio is used in this evaluation. The malignant nodes usually have a long to short axis of less than 2 and appear more round.

Spiculated margins implicate extranodal extension. The diagnostic sensitivity of ultrasonography may be enhanced by colour Doppler. Hilar blood flow pattern is altered in a malignant node. Hypervascularity multiple feeding vessels to the hilum are some of the abnormal features to be noted in a malignant node. When more than one of these features are present lymph node metastasis may be suspected.

The assessment of sentinel node evaluation was initially conceptualised in the early 19705 and this obviated the need for routine lymphadenectomy. This also identified a subset of patients at risk for locoregional spread.7,8

Initially the concept of sentinel node evaluation was done on malignant melanoma with encouraging results. ${ }^{9}$ It was also experimented in other solid tumours including breast cancers.

Various studies have been documented in literature related to SLNB in breast cancers. ${ }^{10}$
The western countries have more advanced studies pertaining to SLNB evaluation in early breast cancers and therapeutics.

Evaluation of SLNB has also been extensively studied in locoregionally advanced breast cancers.

However, the developing countries are yet to catch up with the western centres regarding literature on SLNB.

The standardization, validation of SLNB techniques in breast cancers have certain issues which are yet to be addressed.

Cheaper alternatives to frozen section, gamma - probe methods are yet to be identified and this study in done in an effort to address these issues. ${ }^{11,2}$

In SLNB techniques various studies like Giuliano et al used Isosulfan blue dye (4 $\mathrm{ml}$ of $1 \%$ sigma - Aldrich Milwaukee W1 USA)

This was injected peritumorally at 12, 3, 6 and 9 ' 0 ”' clock position.

To facilitate the absorption of dye centripetal massage was applied around the injected material for few minutes. An oblique incision was made behind the anterior axillary fold after 5 minutes of injecting the dye. The blue lymph vessels where identified by dissection and after identifying the lymph vessels was traced to the blue node distally and primary tumour proximally. The first blue node identified was labelled as sentinel node and removed for analysis. Second node identified was also labelled are removed. Axillary node dissection was then done irrespective of SLNB outcome.

The current study is a descriptive study done to analyse the accuracy between ultrasound guided FNAC of axillary lymph nodes and sentinel lymph node biopsy using methylene blue followed by imprint cytology in clinically node negative early breast cancers.

\section{MATERIALS AND METHODS}

\section{Place of Study}

Meenakshi Mission Hospital and Research Centre.

\section{Duration of Study}

18 months.

\section{Sample Size (n)}

130 patients.

\section{Inclusion Criteria}

All cases of clinically node negative breast cancers undergoing modified radical mastectomy and breast conservation surgery.

\section{Study Method}

Patients are evaluated after taking history, by clinical examination and mammography. FNAC is done in the OPD and patients who fit into the above-mentioned inclusion criteria are selected.

An informed consent is obtained. USG of the breast and axilla is done by a Senior Consultant, Department of Radiology in MMHRC with GE Voluson E8 Ultra sonogram using linear 7-12 MHz probe. Lymph nodes which are round or oval in shape, short axis more than $5 \mathrm{~mm}$ in size are 
selected and with 21-gauge needle FNAC is done. Characters such as fatty hilum (preserved or not) and vascularity (Central or peripheral) are noted.

Under GA, patient in supine position, $4 \mathrm{ml}$ of methylene blue is injected with a 25-gauze needle peritumorally and 1 $\mathrm{ml}$ retroareolarly. After 5 mins. curvilinear skin incision of 5 cms. is given $2 \mathrm{cms}$. below the inferior hair line of the corresponding axilla. Blue stained lymph nodes are identified objectively and are harvested. Haemostasis is achieved with bipolar cautery. This axillary incision is later included in Stewarts incision, for patients undergoing MRM. Mastectomy with axillary clearance is done and nodes are sent for biopsy. For patients undergoing BCS, another suitable incision, apart from axillary incision was made on breast and Breast conservative surgery (BCS) is done.

Two touch imprint smears were taken from 2 cut surface of node respectively. The smear was then air dried for 5-6 minutes and stained with Jenner-Giemsa stain. This Rapid stain had two steps in which Jenner stain was used for 2 minutes and then Giemsa stain used as Counter stain for 7 minutes. The sections where then analysed to look for tumour deposits and where categorised as positive or malignant respectively.

\section{RESULTS}

During the study period from December 2013 to February 2015130 patients who underwent modified Radical mastectomy SLNB and pre-operative axillary evaluation with ultra-sonography were included in the study. In our study the ultrasound evaluation of axillary lymph node metastasis had sensitivity of $80 \%$ and specificity of $40 \%$. Sensitivity of FNAC after methylene blue identified nodes was $99 \%$ and with specificity of $100 \%$. Intra operative imprint cytology assessment of SLN showed a sensitivity of $100 \%$. The final histopathology report was taken as gold standard for analysing the accuracy of results.

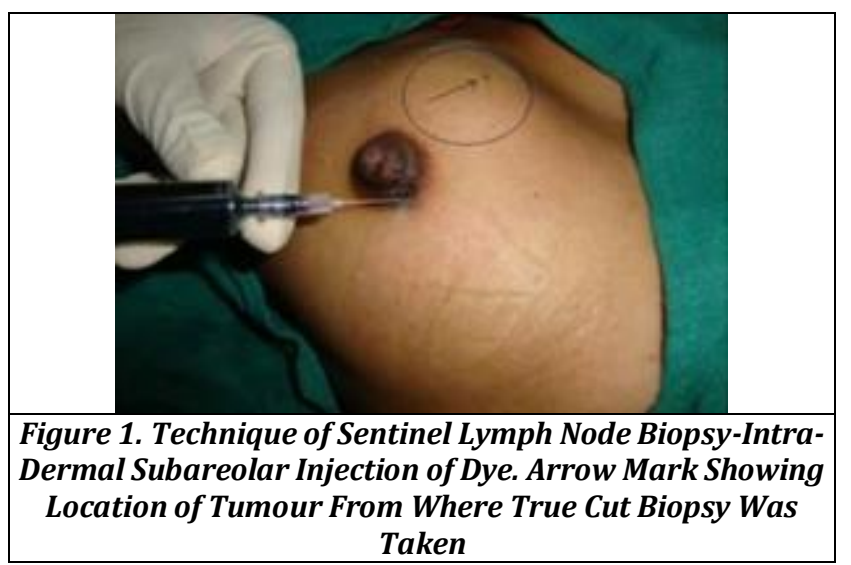

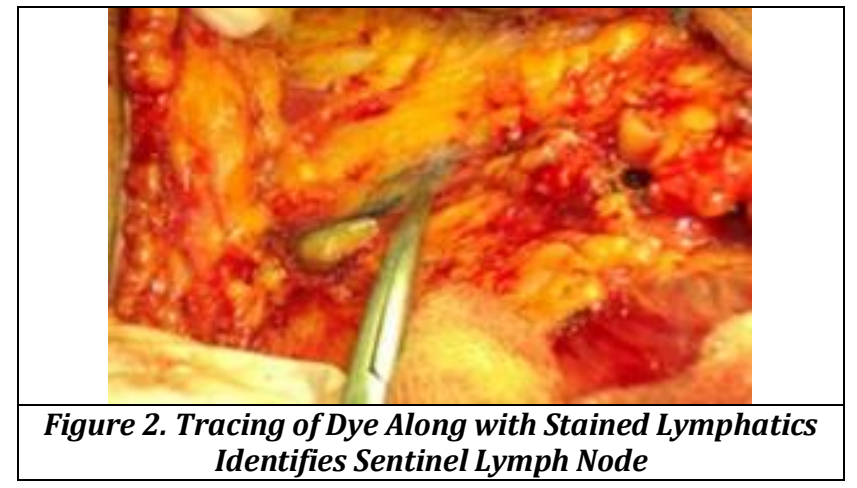
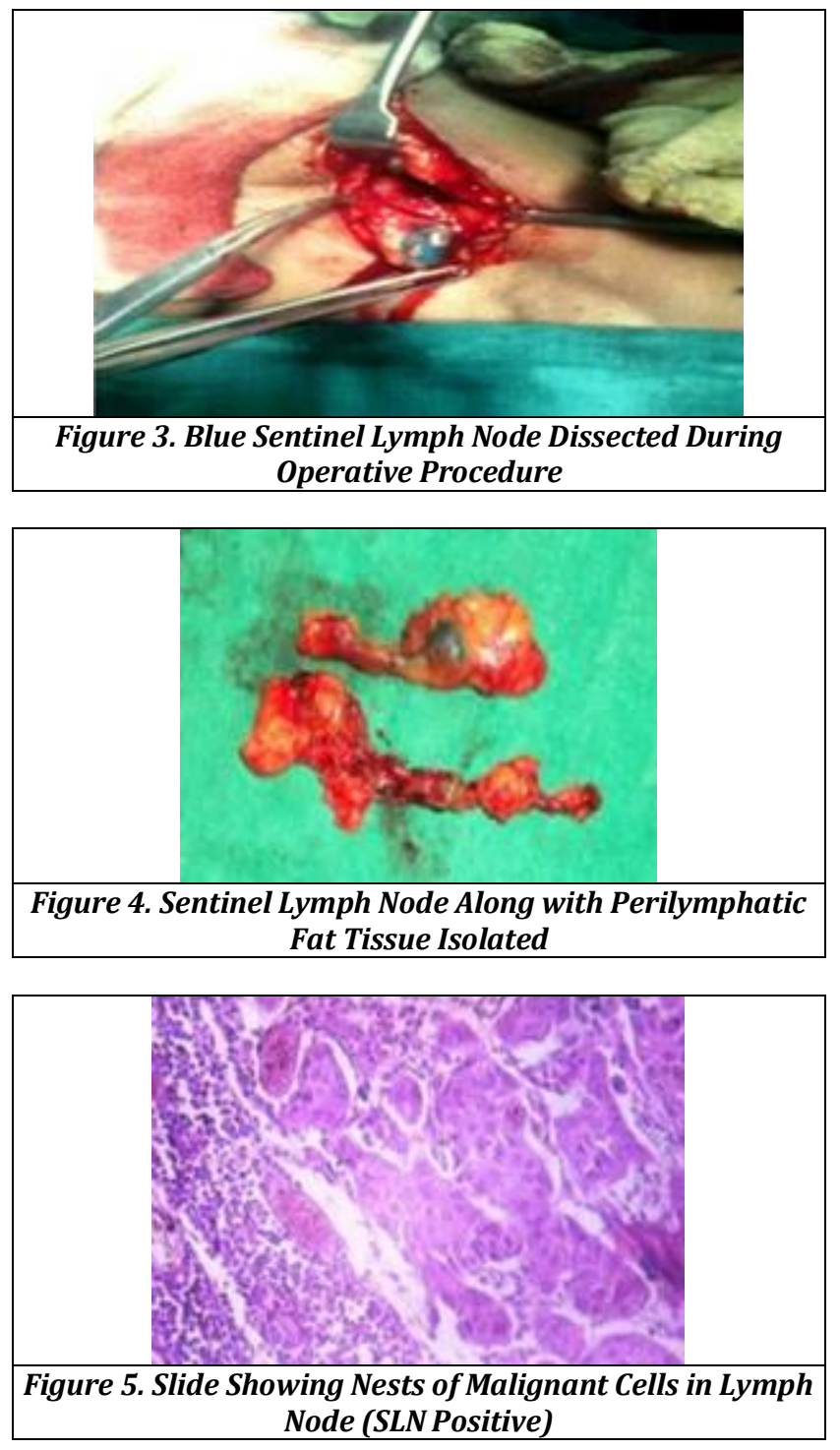

\section{DISCUSSION}

Compared with sentinel node biopsy or axillary node dissection, axillary ultrasonography with FNA cytology is a far less invasive approach to document nodal involvement, involving a simple nonsurgical examination and minimally invasive biopsy. The technique of axillary ultrasonography for breast cancer evaluation was first described in the European literature about 25 years ago. In conjunction with needle biopsy of abnormal - appearing lymph nodes, FNA has been adopted as a diagnostic modality for detection of axillary metastasis. 
In a study done by Marie atherine le et al in which lymph nodes where assessed using ultrasonography and FNAC. In the study it was concluded that both the screening methods where reliable in assessing nodal involvement and to further plan treatment protocols with radiotherapy and chemotherapy. This study also concluded that when compared to lymph node biopsy HPE assessment for metastasis ultrasonography of nodes and FNA from suspicious nodes where equally reliable and simpler minimally invasive technique, then biopsy. The specificity for axillary lymph node ultrasonography in identifying malignancy ranges from $70-96 \%$ sensitivity ranges from 50 $73 \%$. When FNAC is included to evaluate abnormal nodes detected by ultrasonography the specificity then is markedly improved even up to $100 \%$. The identification rate in 38 patients is $82 \%$ with $4 \%$ false negative results.

Surgery is one of the most important modalities of treatment in carcinoma breast. Less invasive methods then SLNB is now a days practiced with mode of curative therapy. Due to a high morbidity of $30-40 \%$ following ALND there is a radical shift in the management of breast cancers which are node negative.

In a study conducted by Popli MB et al.

Which was a prospective study done to assess the feasibility and diagnostic accuracy of pre-operative ultrasound guided FNAC of ALN in patients with carcinoma breast. Thirty breast cancer patients with axillary involvement who were to be taken up for surgery was included in this study. Ultrasound evaluation of nodes to assess lymph node morphology size shape was done. FNAC was then done to confirm USG findings. There findings were evaluated with status of axillary nodes by histopathological examination. Out of 30 patients 8 had T1 disease 16 had T2 disease 5 had T3 disease and one had T4 disease. In their study ultrasound evaluation of ALN had a sensitivity of $86.3 \%$ and specificity of $41.6 \%$. A positive predictive value of $79 \%$ and a negative predictive value of $50 \%$. The diagnostic accuracy of $73.3 \%$ was concluded.

In our study we also had a similar sensitivity and specificity for ultrasound evaluation of ALN.

Sensitivity of USG FNAC was 78-95\% specificity was $100 \%$ positive predictive value of $100 \%$ Negative predictive value was $55.56 \%$ and the diagnostic accuracy was $83.33 \%$. The study concluded that USG - FNAC assessment of ALN is a minimally invasive, cheap early double reliable technique to asses lymph node involvement in breast cancers. When USG FNA was positive in axillary nodes then these cases can be sent for axillary lymph node dissection. When USG - FNAC was negative then a Sentinel node biopsy can be done. By this considerable amount of operating time can be saved and this can be practiced in centres where costly dyes gamma cameras and nuclear medicine facilities are not available. In yet another study by Suryanarayana Deo $^{5}$ et al in which 76 women with breast cancers were evaluated. Here again blue dye was used to asses lymph node status.

Dr. Padmanabha Inamdar et al conducted pilot study of 35 patients of histopathologically diagnosed patients of early breast cancer. Site, amount and quantity of dye injected were noted. The breast tissue along with axillary lymph nodes and sentinel lymph nodes were sent separately for histopathological examination. The presence and absence of metastases in sentinel nodes and axillary nodes were compared. SLN identification rate in our study is $94.2 \%$ with Sensitivity was $100 \%$, Specificity $87.5 \%$, Predictive value of positive test $20 \%$, Predictive value of negative test $100 \%$, false negative rate $0 \%$, false positive rate $12.5 \%$, Overall Accuracy- $87.87 \%$. Nearly $30 \%$ of patients of Breast Cancer could have been saved of unnecessary axillary dissection if sentinel lymph node biopsy technique is used. The study concluded that SLN can be used confidently in patients of early breast cancer and axilla can be spared of unnecessary dissection provided frozen section biopsy is available thereby minimizing morbidity associated with MRM.

Jorien Bonemma et al conducted a study on ultrasound guided aspiration biopsy for detection of non-palpable Axillary node in 150 breast cancer patients and found out that it had a sensitivity of $80 \%$ and specificity of $100 \%$ and detected metastasis in $63 \%$ of node positive patients. They concluded that FNAC is an easy reliable inexpensive method for identifying patients with positive nodes.

Christiane a Voit et al concluded that US-guided FNAC of SNs is highly accurate. Up to $65 \%$ of the patients with SNpositive results in their institution could have been spared an SN procedure. Four hundred consecutive patients who underwent lymphoscintigraphy subsequently underwent US examination before the SN procedure. When the US examination showed a suspicious or malignant pattern, patients underwent an FNAC. Median Breslow thickness was $1.8 \mathrm{~mm}$; mean follow-up was 42 months (range, 4 to 82 months). They considered the US-guided FNAC positive if either US and/or FNAC were positive. If US was suggestive of abnormality, but FNAC was negative, the US-guided FNAC was considered negative. US-guided FNAC identified 51 (65\%) of 79 SN metastases. Specificity was $99 \%$ (317 of 321), with a positive predictive value of $93 \%$ and negative predictive value of $92 \%$.

\section{CONCLUSION}

There is good correlation between pre-operative ultrasound evaluation of nodal status and subsequent methylene blue injected node identification of metastatic deposits.

The FNAC imprint evaluation to identify nodal involvement with histopathology correlation was also very high. Hence these cost-effective techniques play a major role in lymph node evaluation and subsequent surgical management in breast cancers.

Every new therapeutic intervention opens up new vistas and SLN in breast cancer is bringing into focus several treatment strategies which can be tried and tested, as stated by Manishi Bansal et al. The change from Halstedian mastectomy to conservation surgery combined with breast irradiation has benefited thousands of women. This has more to do with staging the axillary nodes than offering any therapeutic benefit and its attendant long-term morbidity is substantial. Management of the axilla thus needs a careful reappraisal in terms of treatment related quality of life and survival. SLN biopsy has been proven to play an important role in staging of the axilla in breast cancer. This provides an opportunity to avoid extensive axillary dissection and also to choose appropriate adjuvant treatment. Patients with early 


\section{Jemds.com}

breast cancer have a good prognosis and, therefore, should receive the best treatment so as to improve survival and quality of life, and decrease long term morbidity.

\section{REFERENCES}

[1] Deo S, Samaiya A, Jain P, et al. Sentinel lymph node biopsy assessment using intraoperative imprint cytology in breast cancer patients: results of a validation study. Asian Journal of Surgery 2004;27(4):294-8.

[2] Giuliano AE Kirgan DM, Guenther JM, et al. Lymphatic mapping and sentinel lymphadenectomy for breast cancer. Ann Surg 1994;220(3):391-8, discussion 398401.

[3] McMasters KM, Wong SL, Tuttle TM, et al. Preoperative lymphoscintigraphy for breast cancer does not improve the ability to identify axillary sentinel lymph nodes. Annals of Surgery 2000;231(5):724-31.

[4] Wong JH, Cagle LA, Morton DL. Lymphatic drainage of skin to a sentinel lymph node in a feline model. Ann Surg 1991;214(5):637-41.

[5] Clarke D, Khonji NI, Mansel RE, et al. Sentinel node biopsy in breast cancer: ALMANAC Trial. World J Surg 2001;25(6):819-22.

\section{Original Research Article}

[6] Rashmi S, Kamala S, Murthy SS, et al. Predicting the molecular subtype of breast cancer based on mammography and ultrasound findings. Indian Journal of Radiology and imaging 2018;28(3):354-61.

[7] Nano MT, Kollias J, Farshid G, et al. Clinical impact of false - negative sentinel node biopsy in primary breast cancer. British Journal of Surgery 2002;89(11):14304.

[8] Motomura K, Inaji H, Komoike $\mathrm{Y}$, et al. Intraoperative sentinel lymph node examination by imprint cytology and frozen sectioning during breast surgery. British Journal of Surgery 2000;87(5):597-601.

[9] Leong SPL. The role of sentinel lymph nodes in malignant melanoma. Surgical Clinics of North America 2000;80(6):1741-57.

[10] Lim RB, Wong JH. Sentinel lymphadenectomy in gynecologic and solid malignancies other than melanoma and breast cancer. Surgical Clinics of North America 2000;80(6):1787-98.

[11] Krag DN, Harlow S, Weaver D, et al. Radiolabeled sentinel node biopsy: collaborative trial with the National Cancer Institute. World J Surg 2001;25(6):823-8. 